\title{
Dynamics of the Crystal to Plastic Crystal Transition in the Hydrogen Bonded $N$-Isopropylpropionamide
}

\author{
Filip Meersman, ${ }^{\dagger}$ Barbara Geukens, ${ }^{\dagger}$ Michael Wübbenhorst,,${ }^{\ddagger}$ Jan Leys,${ }^{\ddagger}$ Simone Napolitano, ${ }^{\ddagger}$ \\ Yaroslav Filinchuk, ${ }^{\S}$ Guy Van Assche," Bruno Van Mele," and Erik Nies*,,$+\perp$ \\ Division of Molecular and Nanomaterials, Department of Chemistry, Katholieke Universiteit Leuven, \\ Celestijnenlaan 200F, B-3001 Leuven, Belgium, Department of Physics and Astronomy, Katholieke Universiteit \\ Leuven, Celestijnenlaan 200D, B-3001 Leuven, Belgium, Swiss-Norwegian Beamlines at the European \\ Synchrotron Radiation Facility, BP-220, F-38043 Grenoble, France, Research Unit Physical Chemistry and \\ Polymer Science, Vrije Universiteit Brussel, Pleinlaan 2, B-1050 Brussels, Belgium, and Laboratory of Polymer \\ Technology, Eindhoven University of Technology, P.O. Box 513, 5600MB Eindhoven, The Netherlands
}

Received: June 1, 2010; Revised Manuscript Received: September 2, 2010

$N$-Isopropylpropionamide (NiPPA), which can self-associate via hydrogen bonds, was found to undergo a solid-solid transition as identified by DSC and X-ray diffraction. Below the melting temperature of $51^{\circ} \mathrm{C}$ NIPPA adopts a plastic crystalline state with a tetragonal unit cell until it transforms into an ordered crystal with a monoclinic structure at temperatures $\leq 10^{\circ} \mathrm{C}$. Dielectric spectroscopy was used to characterize the dynamics of the system, determining the activation parameters for the plastic to crystalline phase transition. The activation enthalpy is relatively high, as expected for a system that involves hydrogen bonds. However, most of the activation energy as the plastic phase assumes a more crystalline state is due to the activation entropy, suggesting that the increased cooperativity observed in the relaxation processes is due to a steric locking of the molecules.

\section{Introduction}

Crystalline solids often undergo structural rearrangements in response to pressure or temperature. ${ }^{1,2}$ These so-called polymorphs have different physical and chemical properties, which can be of interest, for instance, for pharmaceutical applications in the case of small molecules and for thin film devices in the case of crystalline polymers. ${ }^{2,3}$ However, molecules can also be organized in other solid phases, the so-called mesophases, which include plastic crystals and condis crystals. ${ }^{4}$ Plastic crystals are characterized by a crystal-like positional order with local rotational disorder and mobility. A large number of molecules are known to adopt a plastic crystalline state, including cyclohexane, ${ }^{5}$ succinonitrile, ${ }^{6}$ and fullerene. ${ }^{7}$ Even two-component plastic crystals, such as cyclohexanol-neopentylglycol, are known to exist. ${ }^{8}$ Apart from an interest in the understanding of the relations between different (solid) phases, 9,10 plastic crystals are also potentially interesting candidates for the development of fuel cells and solid state thermal energy storage materials. ${ }^{6,8}$ The latter arises from the high enthalpies that are often associated with the ordered crystal to plastic crystal transition. ${ }^{11}$

In the present work we show that $N$-isopropylpropionamide (NiPPA), which can be considered to be the repeat unit of the thermoresponsive water-soluble poly( $N$-isopropylacrylamide) (PNiPA), undergoes an ordered crystal to plastic crystal transition at $10^{\circ} \mathrm{C}$. NiPPA can interact with water as well as selfassociate via hydrogen bonds and is therefore used as a model

* To whom correspondence should be addressed. E-mail: erik.nies@ chem.kuleuven.be.

$\uparrow$ Department of Chemistry, Katholieke Universiteit Leuven.

* Department of Physics and Astronomy, Katholieke Universiteit Leuven.

$\S$ European Synchrotron Radiation Facility.

"Vrije Universiteit Brussel.

${ }^{\perp}$ Eindhoven University of Technology. system for the investigation of the molecular aspects responsible for the observed phase behavior of polymers interacting via hydrogen bonds, such as PNiPA. ${ }^{12}$ As previously pointed out by Hulme et al., not many hydrogen bond forming molecules are known to form plastic crystals. ${ }^{13}$ Presumably this is due to the fact that the hydrogen bonds hinder the molecular rotation. In the particular case of 3-azabicyclo[3.3.1]nonane-2,4-dione, cluster calculations suggested that the hydrogen bond interaction energy might be unusually low. ${ }^{13}$ A weakening of the hydrogen bond strength in the plastic phase has also been deduced from infrared and Raman spectroscopy. ${ }^{11,14}$ For example, it was shown that 2-methyladamantanol forms hydrogen bonded dimers in the ordered crystalline state, whereas in the plastic crystal monomers and dimers are in equilibrium. ${ }^{14}$ Here the ordered and plastic crystalline phases of NiPPA are characterized by $\mathrm{X}$-ray diffraction and dielectric spectroscopy is used to monitor the changes in molecular rotation with temperature. The thermodynamic properties associated with the phase transitions are determined by DSC and the fate of the hydrogen bonds in the solid-solid and solid-liquid transitions is assessed by FTIR spectroscopy.

\section{Experimental Section}

Sample Material. NiPPA was synthesized as described previously. ${ }^{12}$

Differential Scanning Calorimetry. DSC measurements were performed on a TA Instruments Q2000 DSC ( $\mathrm{T}_{\text {zero }} \mathrm{DSC}$ technique) equipped with a RCS cooling accessory and purged

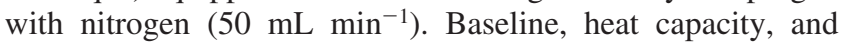
temperature were calibrated with sapphire and indium. TA Instruments $\mathrm{T}_{\text {zero }}$ hermetic aluminum crucibles of $40 \mu \mathrm{L}$ were used. The sample mass was $7 \mathrm{mg}$. The heating/cooling rates applied were 1 and $10{ }^{\circ} \mathrm{C} / \mathrm{min}$. The temperature range studied 
was -90 to $+65^{\circ} \mathrm{C}$. The onset of the melting (crystallization) peak is taken as the melting (crystallization) temperature.

X-ray Diffraction. Angular dispersive diffraction data were collected at the Swiss-Norwegian beamline (BM1A) $(\lambda=$ $0.7183 \AA$ ) at the European Synchrotron Radiation Facility (ESRF) (Grenoble, France) using MAR345 image plate detector. The sample-to-detector distance and the image plate tilt angles were calibrated using a crystalline $\mathrm{Si}$ standard. The sample was enclosed in an aluminum DSC pan and placed in a Linkam hot stage. The temperature was varied from -30 to $+20{ }^{\circ} \mathrm{C}$, with an increment of $0.5{ }^{\circ} \mathrm{C} / \mathrm{min}$. The two-dimensional diffraction images were azimuthally integrated using the ESRF Fit2D program, ${ }^{15}$ yielding one-dimensional intensity $(I)$ versus $2 \theta$ (where $2 \theta$ is the diffraction angle $0^{\circ}<2 \theta<30^{\circ}$ ).

Fourier Transform Infrared Spectroscopy. Infrared spectra were recorded on a Bruker IFS66 FTIR spectrometer equipped with a liquid nitrogen cooled MCT detector at a nominal resolution of $2 \mathrm{~cm}^{-1}$. Each spectrum is the result of the accumulation and averaging of 256 interferograms. The sample compartment was continuously purged with dry air to minimize the spectral contribution of atmospheric water.

Solid NIPPA was sandwiched between two $\mathrm{CaF}_{2}$ windows located in a temperature cell placed in a heating jacket controlled by a Graseby Specac (Orpington, U.K.) automatic temperature controller. The sample was first melted at $55^{\circ} \mathrm{C}$ and subsequently slowly cooled to $-14{ }^{\circ} \mathrm{C}$ and left at this temperature for $2 \mathrm{~h}$ prior to heating to $65^{\circ} \mathrm{C}$. Temperature scans were made at a rate of $0.2{ }^{\circ} \mathrm{C} \mathrm{min}{ }^{-1}$. NiPPA was dissolved at $5 \mathrm{wt} \%$ in dimethyl sulfoxide (DMSO) (BDH Laboratory Suppliers) and measured between two $\mathrm{CaF}_{2}$ windows separated by a $50 \mu \mathrm{m}$ spacer at $25{ }^{\circ} \mathrm{C}$. A linear baseline correction was made in the carbonyl stretching region $\left(\sim 1580-1720 \mathrm{~cm}^{-1}\right)$.

Dielectric Spectroscopy. Dielectric measurements at frequencies from $0.1 \mathrm{~Hz}$ to $10 \mathrm{MHz}$ were performed by a Novocontrol Alpha analyzer in combination with a Novocontrol Quatro temperature controller and cryo-system. The sample was heated well above the melting temperature, and together with quartz fibers, acting as spacers, placed in a sealed parallel plate capacitor with brass electrodes of $20 \mathrm{~mm}$ diameter. The thickness of the sample was $50 \mu \mathrm{m}$. Dielectric spectra were acquired isothermally at temperatures between +110 and -120 ${ }^{\circ} \mathrm{C}$ in steps of $2.5 \mathrm{~K}$. This temperature scheme resulted in an effective cooling rate of $0.5{ }^{\circ} \mathrm{C} / \mathrm{min}$.

The high frequency spectra were measured with a HewlettPackard HP 4291B Impedance Analyzer, operating between 1 $\mathrm{MHz}$ and $1 \mathrm{GHz}$. A custom-made parallel plate measurement cell was used. Temperature was controlled by a fluid flow and its stability was on the order of $0.05 \mathrm{~K}$. Spectra were acquired in steps of $1 \mathrm{~K}$ after 25 min of stabilization.

\section{Results and Discussion}

Plastic Crystalline to Ordered Crystal Transition of NiPPA. When characterizing the neat NiPPA by DSC, we observed two endothermic transitions at 10 and $51{ }^{\circ} \mathrm{C}$, respectively (Figure 1). The latter transition corresponds to the melting of the crystalline NiPPA. To characterize the low temperature transition, NiPPA was heated from -30 to $+20{ }^{\circ} \mathrm{C}$ and its structural features were monitored by powder wide-angle $\mathrm{X}$-ray diffraction. This is shown in Figure 2, where it can be seen that several diffraction peaks change in intensity, indicating a transformation between two different crystalline structures. The largest changes are observed for the reflections at $2 \theta=5.10$ and $6.38^{\circ}$ that appear or disappear completely.

Powder patterns at $+4{ }^{\circ} \mathrm{C}$ and $+13{ }^{\circ} \mathrm{C}$ were selected as representative of the low and high temperature phases, respec-

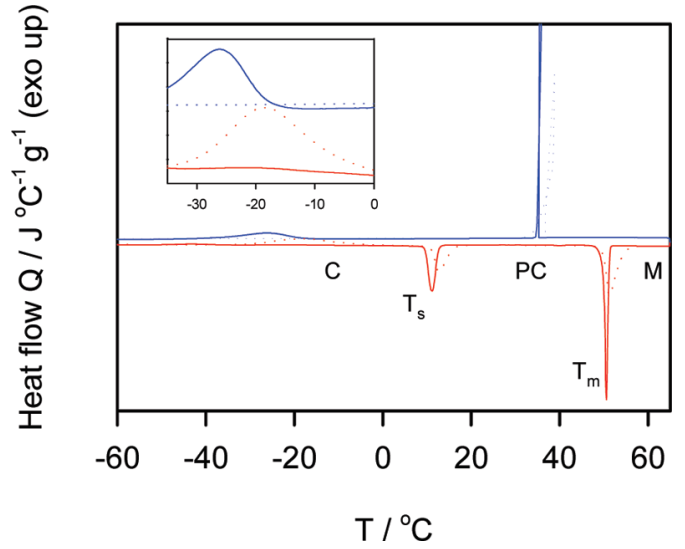

Figure 1. DSC thermograms of the neat NiPPA sample measured at a rate of $1{ }^{\circ} \mathrm{C} / \mathrm{min}$ (solid line) and $10{ }^{\circ} \mathrm{C} / \mathrm{min}$ (dotted line), during cooling (blue curve) and heating (red curve). The inset shows a magnification of the region between -35 and $0{ }^{\circ} \mathrm{C}$, where the transformation between the crystal (C) and the plastic crystal (PC) takes place. The melt (M) phase is also indicated.

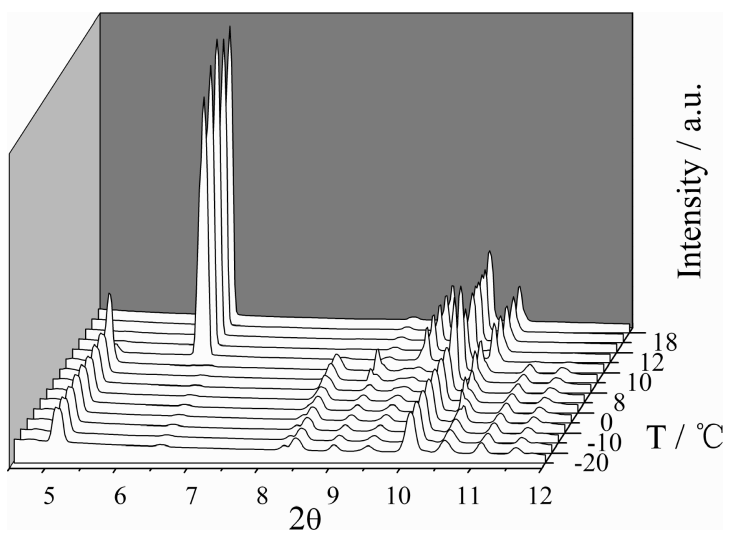

Figure 2. Changes in the powder diffraction pattern of the solid NiPPA sample between -30 and $+20{ }^{\circ} \mathrm{C}$. The wavelength of the radiation was $\lambda=0.7183 \AA$.

tively, and further used for indexing and solving the structure. At $+4{ }^{\circ} \mathrm{C} 14$ diffraction peaks were indexed by Dicvol ${ }^{16}$ in a primitive monoclinic cell ( $a=17.4621(16) \AA, b=8.9077(13)$ $\AA, c=4.9399(5) \AA)$. Systematic absences indicated the space group $P 2{ }_{1} / a$ (Figure 3 ). The structure was resolved in this space group by global optimization in direct space using the program FOX,${ }^{17}$ varying the position, orientation, and conformation of one independent molecule located in the general position. Fifteen geometry restraints were imposed on interatomic distances and angles during optimization. Including the modeling of the preferred orientation in the global optimization did not change the resulting structure but improved the fit. ${ }^{18}$ One parameter was optimized (March-Dollase model), with the [110] direction of the preferred orientation. The crystal symmetry of the resulting structure is very reasonable: hydrocarbon chains are grouped together, and $\mathrm{C}=\mathrm{O}$ and $\mathrm{N}-\mathrm{H}$ groups form a hydrogen bond at a $\mathrm{N} \cdots \mathrm{O}$ distance of $2.9 \AA$ extending along the $c$ axis. Examination of the resulting structure and analysis by Platon ${ }^{19}$ did not find any higher crystallographic symmetry. The final refinement was done by the Rietveld method using the program Fullprof. ${ }^{20}$ The background was described by linear interpolation between selected points. The preferred orientation was modeled by one parameter (March's function), which converged to the value 1.17(1). Coordinates of eight non-hydrogen atoms and an overall atomic displacement factor were refined. Seven restraints on interatomic distances and six on angles were 

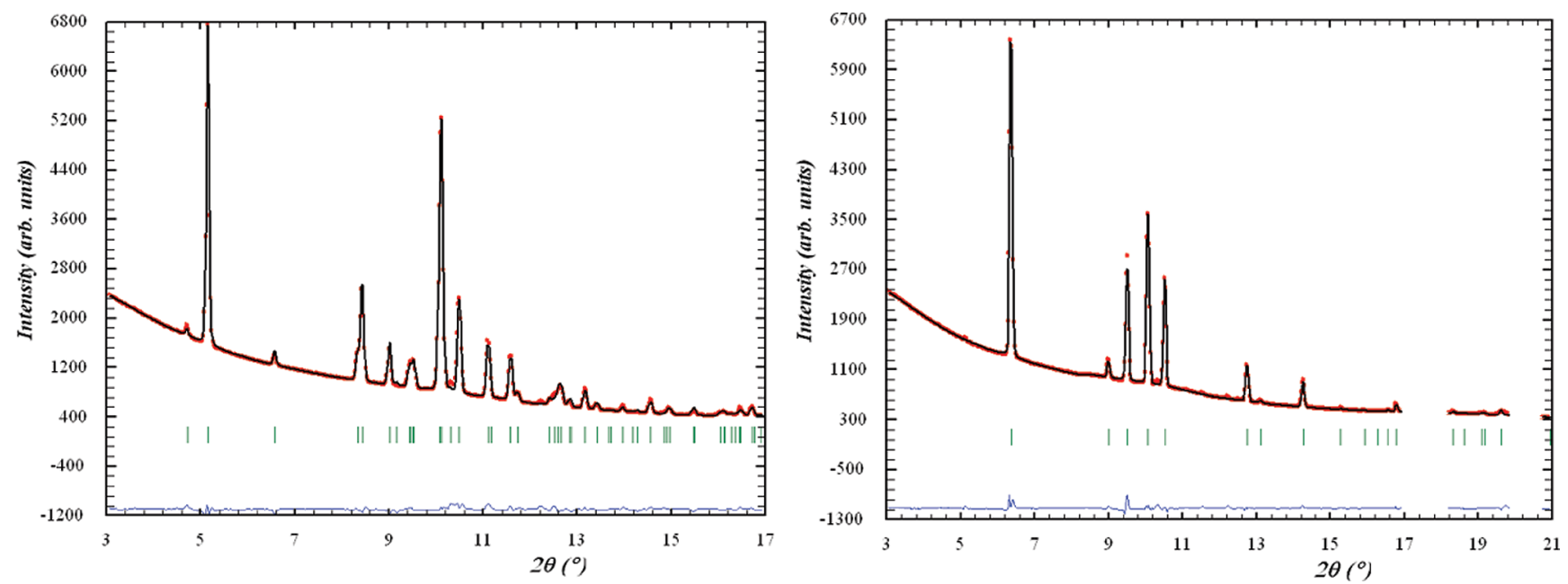

Figure 3. Rietveld refinement profile for the low-temperature phase at $4{ }^{\circ} \mathrm{C}$ (left) and the le Bail fit for the high-temperature phase at $13{ }^{\circ} \mathrm{C}$ (right), $\lambda=0.7183 \AA$.
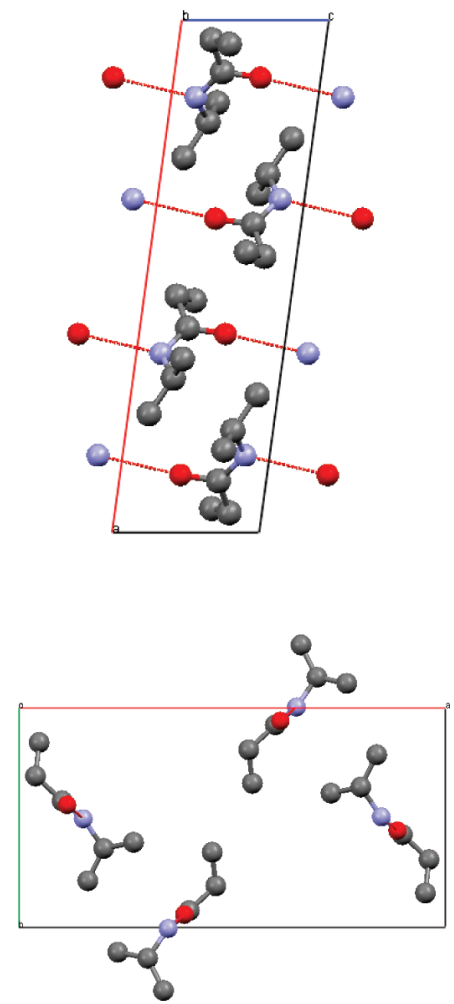

Figure 4. Crystal structure of NiPPA at $4{ }^{\circ} \mathrm{C}$. The dashed lines are hydrogen bonds between NiPPA molecules. The structure is viewed along the $b$-axis (top) and $c$-axis (bottom) of the monoclinic unit cell.

imposed, using the quantum-chemical geometrical optimization of the free molecule with the program HyperChem, Release 6.0 (Hypercube Inc., Gainesville, FL, U.S.). Hydrogen atoms were omitted in the refinement. The refinement converged at $R_{\mathrm{B}}=$ $6.6 \%, R_{\mathrm{F}}=7.9 \%, R_{\mathrm{p}}=10.1 \%$, and $R_{\mathrm{wp}}=8.7 \%$. The structure of the low temperature phase is illustrated in Figure 4.

In the pattern of the high temperature $\left(+13{ }^{\circ} \mathrm{C}\right)$ phase eight diffraction peaks were indexed by $\operatorname{Dicvol}^{16}$ in a primitive tetragonal cell of nearly twice the smaller volume of the lowtemperature $P 2_{1} / a$ structure. Analysis of systematic absences using the program Chekcell ${ }^{21}$ suggested three possible space groups: $P 4_{2} n m, P \overline{4} n 2$, and $P 4_{2} / m n m$. The groups-subgroup relations between the tetragonal and the monoclinic cells suggest that the high-temperature phase contains only two molecules per unit cell, and thus the structure must be highly disordered. Structure solution in FOX did not yield any reasonable model. Therefore, only a le Bail fit was performed for the hightemperature phase using the program Fullprof. ${ }^{20}$ The refinement converged at $R_{\mathrm{p}}=12.1 \%$ and $R_{\mathrm{wp}}=8.5 \%$. The Rietveld refinement profile is shown in Figure 3.

The reduction in the number of peaks in the powder pattern above $10{ }^{\circ} \mathrm{C}$ reflects the transition to a higher symmetry structure. Taken together, these observations suggest that the high temperature phase observed between 10 and $51{ }^{\circ} \mathrm{C}$ corresponds to a plastic crystalline state. Although plastic crystals often have a cubic symmetry, ${ }^{9,13}$ examples with other types of symmetry exist, including dimethylacetylene and 2-adamantanone. ${ }^{9,22}$ The tetragonal symmetry suggests the presence of some restriction on the rotational freedom of the molecule, as a completely free rotation would result in a cubic symmetry. ${ }^{10}$

The identification of the plastic crystalline phase by X-ray diffraction is corroborated by the DSC analysis. Plastic crystal formation is generally accompanied by a low fusion entropy ( $\left.\Delta S_{\text {fus }}\right)$ and the ratio $\Delta S_{\text {trs }} / \Delta S_{\text {fus }}$ is often, but not always, $\geq 1 .^{13,14}$ For NiPPA the corresponding values are $\Delta S_{\text {trs }}=17.84 \mathrm{~J} \mathrm{~K}^{-1}$ $\mathrm{mol}^{-1}$ and $\Delta S_{\text {fus }}=24.04 \mathrm{~J} \mathrm{~K}^{-1} \mathrm{~mol}^{-1}$, with $\Delta S_{\text {trs }} / \Delta S_{\text {fus }}=0.731$. These values are close to those of the typical plastic crystal adamantane with $\Delta S_{\text {trs }}$ and $\Delta S_{\text {fus }}$ being 16.5 and $23.7 \mathrm{~J} \mathrm{~K}^{-1}$ $\mathrm{mol}^{-1}$, respectively. ${ }^{22,23}$

Stability and Kinetics of Transformation of the Different Phases. The kinetics of the formation of the ordered crystal (C) and plastic crystal (PC) were assessed by investigating the cooling rate dependence of the solid-solid transition by DSC (Figure 1). A cooling rate of $1{ }^{\circ} \mathrm{C} / \mathrm{min}$ results in a solid-solid transformation occurring in the cooling (blue) curve, while for a cooling rate of $10^{\circ} \mathrm{C} / \mathrm{min}$ the solid-solid transformation from the $\mathrm{PC}$ state into the $\mathrm{C}$ state is only visible in the subsequent heating (red) curve (inset Figure 1). When cooling from +65 to $-90{ }^{\circ} \mathrm{C}$, the liquid NiPPA sample will crystallize at around $+36{ }^{\circ} \mathrm{C}$ into the PC state. This crystallization process is accompanied by a release of heat, which in the dynamic DSC experiment results in a slightly increased sample temperature. This phenomenon can be seen in Figure 1 as a loop in the cooling curve at the crystallization temperature $\left(T_{\mathrm{c}}\right)$. At a rate of $10{ }^{\circ} \mathrm{C} / \mathrm{min}$ the $\mathrm{PC}$ state does not transform into the $\mathrm{C}$ state, which is the thermodynamically stable state at lower temperatures, during the time needed to complete the cooling process. 


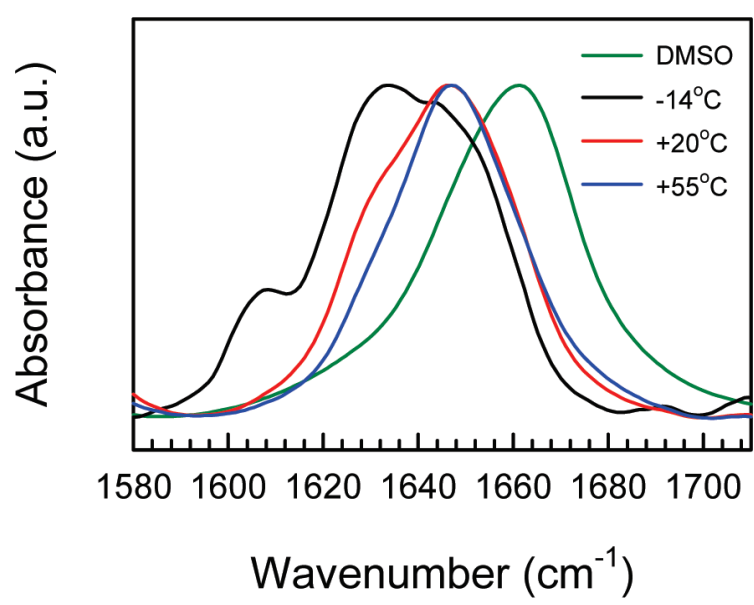

Figure 5. FTIR spectra of the carbonyl stretching region of NiPPA. Spectral intensity was normalized for ease of comparison.

However, in the DSC experiment depicted in Figure 1 the transformation from the $\mathrm{PC}$ state to the $\mathrm{C}$ state happens in the subsequent heating step at around $-35^{\circ} \mathrm{C}$. The transformation from the $\mathrm{PC}$ to the $\mathrm{C}$ state occurs also when waiting long enough at a temperature below the $\mathrm{PC}$-to-C transition temperature. On the other hand, when a cooling rate of $1{ }^{\circ} \mathrm{C} / \mathrm{min}$ is used, the PC state transforms into the $\mathrm{C}$ state at about $-15^{\circ} \mathrm{C}$. In both cases heating results in the solid-solid transformation from the $\mathrm{C}$ state into the PC state at $+10^{\circ} \mathrm{C}$, followed by the melting of the PC state at $+51{ }^{\circ} \mathrm{C}$.

Hydrogen Bonds Remain Intact in the Plastic and Liquid Phase. FTIR spectroscopy was used to investigate whether or not the solid-solid transition is accompanied by a loss of hydrogen bonding. The carbonyl stretching region (1580-1720 $\mathrm{cm}^{-1}$ ) of NiPPA in its PC, C, and liquid states is shown in Figure 5. The spectrum of NiPPA in the aprotic solvent DMSO is also included for comparison. The $\mathrm{C}$ state is characterized by two bands of almost equal intensity around 1647 and $1633 \mathrm{~cm}^{-1}$, as well as an additional weak peak at $1608 \mathrm{~cm}^{-1}$. The complexity of the carbonyl band in the ordered crystal most likely arises from the crystal field effect that is often observed in vibrational spectra of molecular crystals. ${ }^{24,25}$ The $1608 \mathrm{~cm}^{-1}$ peak is absent in the plastic phase, which is characterized by an intense band around $1647 \mathrm{~cm}^{-1}$ and a shoulder at $1633 \mathrm{~cm}^{-1}$. This change in intensity ratio has been observed previously for $\mathrm{N}$-methylacetamide (NMA) ${ }^{26}$ In the liquid state the main peak maximum is also found at $1647 \mathrm{~cm}^{-1}$. In DMSO, on the other hand, the carbonyl stretching band shifts to $1661 \mathrm{~cm}^{-1}$, typical of free, non-hydrogen bonded carbonyl vibrations. This implies that hydrogen bonds are present between NiPPA molecules in both solid phases and the liquid phase, despite differences in molecular arrangement. This is consistent with the findings for NMA. ${ }^{27}$

Dynamics of the Solid-Solid Transition. Further insight into the C-to-PC transition was obtained from dielectric spectroscopy. The strength and frequency dependence of the dielectric response is expressed by the complex dielectric permittivity $\varepsilon^{*}(f)=\varepsilon^{\prime}(f)-\mathrm{i} \varepsilon^{\prime \prime}(f)$, which accounts for both an in-phase capacitive term $\left(\varepsilon^{\prime}\right)$ and a contribution $\varepsilon^{\prime \prime}$ related to dissipation of energy due to electrical conduction and internal friction of molecular dipoles. Measuring the spectral dielectric response thus yields information about the molecular dipolar reorientation mechanisms and their temperature dependence in different crystalline states. ${ }^{28-30}$

Dielectric spectra $\varepsilon^{*}(f)$ consisting of the permittivity $\varepsilon^{\prime}(f)$ and the loss $\varepsilon^{\prime \prime}(f)$ curves, were analyzed using two strategies, the combination of which enables the separation of multiple relaxation processes as well as ohmic conduction in an effective way:

(1) Elimination of the Loss by Ohmic Conductivity. Strong ionic conduction, which usually shows up above the glasstransition temperature, $T_{\mathrm{g}}$, often obscures loss peaks of dipolar origin. The ohmic conduction, scaling as $\varepsilon_{\text {cond }}^{\prime \prime}=\sigma / \varepsilon_{0} \omega(\sigma=$ conductivity, $\varepsilon_{0}=$ vacuum permittivity, $\omega=$ angular frequency $=2 \pi f$ ), might hide relaxation processes in the low frequency region. A convenient technique to eliminate this contribution from $\varepsilon^{\prime \prime}(f)$ is based on the calculation of the derivative of the real part of the dielectric function, which yields an expression for the dielectric loss free from the contribution of the ohmic conduction (eq 1). ${ }^{31}$

$$
\varepsilon_{\text {deriv }}^{\prime \prime}=-\frac{\pi \partial \varepsilon^{\prime}(\omega)}{2 \partial \ln \omega} \approx \varepsilon^{\prime \prime}
$$

The approximation $\varepsilon_{\text {deriv }}^{\prime \prime} \approx \varepsilon^{\prime \prime}$ holds for broad peaks like those of the dynamic glass transition or secondary relaxations. Peaks in spectra obtained via eq 1 are markedly narrower than the measured ones, leading to a better resolution of the overlapping molecular processes.

(2) Least Squares Fitting of Relaxation Spectra. For a quantitative analysis the loss spectra were fitted with a Havriliak-Negami (HN) relaxation function (eq 2), using the Levenberg-Marquardt algorithm:

$$
\varepsilon^{\prime \prime}=-\sum_{k=1}^{n} \operatorname{Im}\left\{\frac{\Delta \varepsilon_{k}}{\left(1+\left(\mathrm{i} \omega \tau_{k}\right)^{a_{k}}\right)^{b_{k}}}\right\}+\frac{\sigma}{\varepsilon_{\mathrm{o}} \omega}
$$

where $\Delta \varepsilon_{k}$ and $\tau_{k}$ denote the relaxation strength and the mean relaxation time of the $k$ th process. The two shape parameters $a_{k}$ and $b_{k}$, which determine the slope of the low frequency loss tail $a_{k}$ and the high frequency loss tail $-a_{k} \cdot b_{k}$, are determined by the underlying distribution in relaxation times. The second term in eq 2 accounts for ohmic conduction.

A fit of the derivative-based loss spectra $\varepsilon_{\text {deriv }}^{\prime \prime}(f)$ with the analytical derivative of the Havriliak-Negami function $\partial \varepsilon_{\mathrm{HN}}^{\prime} / \partial$ $\ln f$ combines the advantages of both approaches resulting in a more precise calculation of the HN-parameters and a highly resolving peak analysis. ${ }^{31}$ The expression for $\partial \varepsilon_{\mathrm{HN}}^{\prime} / \partial \ln \omega$ reads

$$
\frac{\partial \varepsilon_{\mathrm{HN}}^{\prime}}{\partial \ln \omega}=-\frac{a b \Delta \varepsilon(\omega \tau)^{a} \cos \left[a \pi / 2-(1+b) \theta_{\mathrm{HN}}\right]}{\left[1+2(\omega \tau)^{a} \cos (\pi a / 2)+(\omega \tau)^{2 a}\right]^{1+b / 2}}
$$

with

$$
\theta_{\mathrm{HN}}=\arctan \left[\sin (\pi a / 2) /\left((\omega \tau)^{-a}+\cos (\pi a / 2)\right)\right]
$$

The temperature dependence of $\varepsilon_{\text {deriv }}^{\prime \prime}(10 \mathrm{kHz})$ upon heating and cooling is given in Figure 5. This quantity is sensitive to changes in the friction of dipoles at the molecular scale and consequently can be used to monitor structural rearrangements occurring during a phase transition. Two thermal transitions appearing as sharp changes of $\varepsilon_{\text {deriv }}^{\prime \prime}$ correspond to those observed by DSC (Figure 1). The relaxation time $\tau$ and the relaxation strengths $\Delta \varepsilon$ for the different relaxation processes reported in Table 1 are a result of the analysis of the data in Figure 6 by means of 
TABLE 1: Activation Parameters for Dielectric Relaxation Processes in NiPPA

\begin{tabular}{ccccccc}
\hline $\begin{array}{c}\text { relaxation } \\
\text { process }\end{array}$ & phase & $\begin{array}{c}E_{\mathrm{a}}(\mathrm{kJ} \\
\left.\mathrm{mol}^{-1}\right)\end{array}$ & $\log \left(\tau_{\infty}\right)$ & $\begin{array}{c}\Delta H_{\mathbf{0}} \\
\left(\mathrm{kJ} \mathrm{mol}^{-1}\right)\end{array}$ & $\begin{array}{c}\Delta S^{\#}(\mathrm{~kJ} \\
\left.\mathrm{mol}^{-1} \mathrm{~K}^{-1}\right)\end{array}$ & $\Delta \varepsilon$ \\
\hline$\sigma$ & M & 27.85 & -6.69 & & & \\
L & M & 33.95 & -14.12 & 30.78 & 0.02 & 45.0 \\
I & PC & 78.94 & -15.63 & 65.99 & 0.05 & 0.1775 \\
II & PC & 70.90 & -16.13 & 57.06 & 0.06 & 0.0525 \\
III & PC & 75.78 & -18.29 & 53.33 & 0.10 & 0.0190 \\
L & PC & 28.87 & -13.62 & 27.08 & 0.02 & 0.3500 \\
I & C & 89.44 & -16.33 & 71.59 & 0.06 & 2.0250 \\
II & C & 83.57 & -18.24 & 59.21 & 0.10 & 0.0775 \\
III & C & 71.79 & -20.14 & 45.44 & 0.14 & 0.0016 \\
L & C & 45.04 & -16.86 & 33.99 & 0.08 & 0.0350
\end{tabular}

eqs 3 and 4 . An example of deconvoluted spectra collected in the plastic crystalline phase is shown in Figure 7, which also shows the temperature dependence of the relaxation times of processes, in a cooling scan for the low frequencies and a heating ramp for the high-frequency setup. Except for the values of the transition temperatures, the analysis of the molecular dynamics presented in the following paragraphs does not depend on the experimental scanning conditions. To facilitate a quantitative treatment of the results, we choose to present the data set with the highest signal-to-noise ratio. All observable relaxation processes can be described by the Arrhenius equation (eq 5):

$$
\tau(T)=\tau_{\infty} \exp \left(E_{\mathrm{a}} / R T\right)
$$

where $E_{\mathrm{a}}$ is the activation energy and the parameter and $\tau_{\infty}$ represents the ultimate relaxation time in the limit $T \rightarrow \infty$. Above $T_{\mathrm{m}}$, a fast relaxation mode $\left(\tau_{\mathrm{L}}\right)$ appearing in the megahertz range

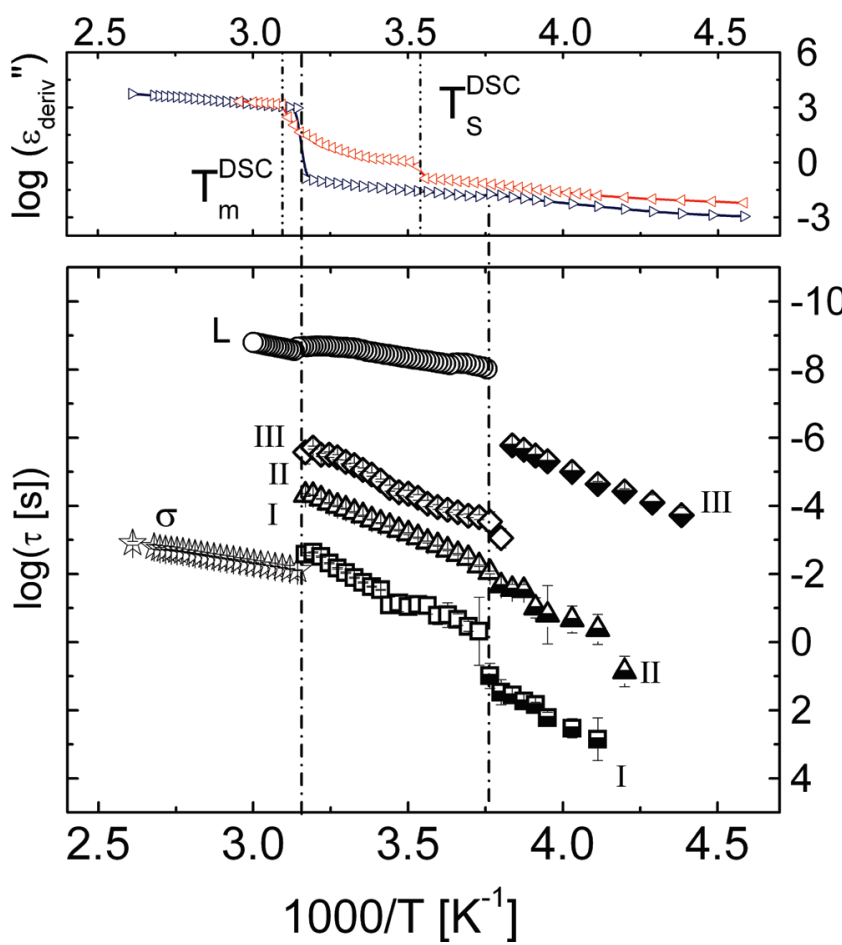

Figure 6. Determination of the molecular dynamics by dielectric spectroscopy. Top: conduction-free loss ( $\left.\varepsilon_{\text {deriv }}^{\prime \prime}\right)$ of NiPPA during cooling from +100 to $-100{ }^{\circ} \mathrm{C}$ (blue arrows) and subsequent heating (red arrows). Bottom: Arrhenius plot for NiPPA showing the log of the relaxation time $\tau$ as a function of the reciprocal temperature for all dielectric processes in the liquid phase, the plastic crystalline, and the crystalline phase: $\mathrm{MHz}(\mathrm{O}), \sim 100 \mathrm{kHz}(\mathbf{\Delta}), \mathrm{kHz}(\boldsymbol{\nabla})$, and $\mathrm{Hz}$ range $(\square)$. The solid-solid transition $\left(T_{\mathrm{s}}\right)$ and melting $\left(T_{\mathrm{m}}\right)$ temperatures are indicated.

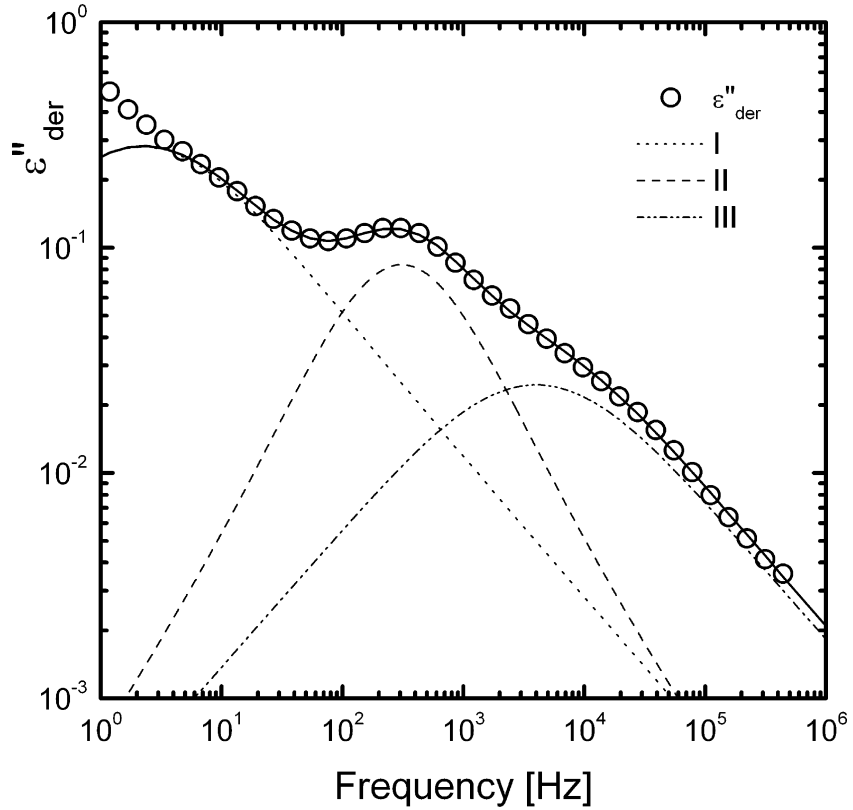

Figure 7. Deconvoluted dielectric spectrum of crystalline NiPPA at $15{ }^{\circ} \mathrm{C}$.

dominates the liquid dynamics. The same relaxation mode imposes the activation energy of a slow relaxation process $(\sigma)$ appearing at lower frequencies. This last process is the direct manifestation of the conductivity of the material, and its intensity drops by several orders of magnitude upon crystallization. Below $T_{\mathrm{m}}$, three dielectric relaxation processes are found. These processes, which are denoted by I, II, and III, respectively at low (hertz range), intermediate (kilohertz range), and high frequencies $(\sim 100 \mathrm{kHz})$, correspond to the dynamics in the (plastic) crystalline phase. The observed splitting into individual molecular relaxation processes can be taken as a measure for the increase in the degree of anisotropy in molecular motions. When passing from the plastic crystalline to the crystal state, both the peak separation and the mean activation energy (the effective rotational barrier height) further increase. Those observations are consistent with our picture based on an increase of degree of molecular order upon cooling from the PC state to the $\mathrm{C}$ state.

The appearance of a residual component of $\tau_{\mathrm{L}}$, with a dielectric strength on the order of $1 \%$ of the value in the liquid state, suggests the presence of liquid-like defects in the crystalline state. The strong reduction of $\Delta \varepsilon$ for the faster motions (high frequency processes) both at $T_{\mathrm{m}}$ and at $T_{\mathrm{s}}$ are related to a reduction of the mean square dipole moment $\left(\left\langle\mu^{2}\right\rangle\right)$. The restrictions to molecular motion, imposed by a more efficient packing in the more ordered phases, strongly limit the value of the solid angle that can be explored by the relaxing units in their microenvironment and thus the value of $\left\langle u^{2}\right\rangle$. Less intuitive is the trend of the dielectric strength for the slower processes I and II at $T_{\mathrm{s}}$. The increase of $\Delta \varepsilon$ is here probably caused by a perturbation of the Kirkwood correlation factor $(g)$, expressing the reciprocal influence among neighboring molecules. The decrease in symmetry passing from PC to $\mathrm{C}$, as evidenced by the increase in the number of reflection peaks in XRD, would favor collective motions resulting in higher $g$ values. Further evidence on this observation was gained by using an approach proposed by Eyring and revised by Starkweather. From the experimental activation energy $E_{\mathrm{a}},{ }^{32}$ it was possible to obtain the activation entropy $\left(\Delta S^{\#}\right)$ and activation enthalpy $\left(\Delta H^{\sharp}\right)$ at the temperature $T^{\prime}$, which is the temperature of the 
maximum of the relaxation peak at a chosen reference frequency $f(f=1 \mathrm{~Hz})$,

$$
\begin{gathered}
\Delta S^{\#}=\frac{1}{T^{\prime}}\left[E_{\mathrm{a}}-R T^{\prime}\left(1+\ln \frac{k_{\mathrm{B}} T^{\prime}}{2 \pi h f}\right)\right] \\
\Delta H^{\#}=E_{\mathrm{a}}-R T^{\prime}
\end{gathered}
$$

here $k_{\mathrm{B}}$ is the Boltzmann constant and $h$ is the Planck constant. The calculated activation parameters are given in Table 1 . In Starkweather's approach, the value of $\Delta H^{\#}$ is a measure for the cooperativity of the motions, with cooperative motions characterized by an activation enthalpy larger than $\Delta H_{0}$ that is the theoretical activation enthalpy associated with zero activation entropy, calculated as

$$
\Delta H_{0}=R T^{\prime}\left(1+\ln \frac{k_{\mathrm{B}} T^{\prime}}{2 \pi h f}\right)
$$

A similar criterion can be defined for $\Delta S^{\#}$, relating the cooperative character of a process to a nonzero value of the activation entropy. As a result of this analysis, the relaxation processes not attributed to a liquid-like phase are characterized by positive values of the activation entropy, indicating the cooperative nature of the underlying molecular dynamics. In particular, the slower processes I and II, for which $\Delta \varepsilon$ increases when passing form the plastic crystalline to the crystalline phase, are those characterized by the highest value of $\Delta S^{\#}$ and thus a larger cooperative component.

Moreover, the analysis provided by the Starkweather approach suggests that the increase in activation energy $E_{\mathrm{a}}$ at $T_{\mathrm{s}}$ is caused by a further excess of activation entropy. The relative changes in the activation enthalpy are 1-2 orders of magnitude smaller than those in $\Delta S^{\#}$ (Table 1). A similar phenomenon was previously observed for the mesophase-crystalline transition of poly[bis(trifluorethoxyphosphazene) $]^{29}$ and would imply that at $T_{\mathrm{s}}$ the relaxation processes gain part of their high cooperativity due to a steric interlocking of the molecules.

\section{Conclusions}

Although the existence of rotating molecules in a crystal was already proposed in the 1920s and a large number of plastic crystals have subsequently been identified since, the number of plastic crystals in which hydrogen bonds exist remains relatively small. ${ }^{9,13}$ Here we identified and characterized a new plastic crystal phase formed by $N$-isopropylpropionamide that occurs between 10 and $51{ }^{\circ} \mathrm{C}$. NiPPA has various rotational degrees of freedom, including rotation around its center of mass. However, as the structure of the plastic phase is not completely resolved, it is not possible to univocally assign any relaxation times $\tau_{\mathrm{i}}$ to a specific degree of freedom.

Typical activation enthalpies for non-hydrogen bonding small organic molecules are $\leq 12 \mathrm{~kJ} \mathrm{~mol}^{-1} \cdot 33,34$ In the case of DL-isoborneol the presence of a hydrogen bond interaction was reflected in an increased activation enthalpy of $\sim 22 \mathrm{~kJ} \mathrm{~mol}^{-1}$. $^{33}$ Therefore, the relatively high activation enthalpies observed in this work are as expected for a system that involves multiple hydrogen bonds. Interestingly, most of the increase in activation energy as the plastic phase adopts a more crystalline state is due to an excess of the activation entropy. This suggests that the increased cooperativity observed in the relaxation processes is due to steric locking of the molecules and that the hydrogen bonds are preserved in the plastic crystalline phase. The latter is confirmed by FTIR spectroscopy.

Acknowledgment. We are grateful to the Swiss-Norwegian Beamlines at the ESRF for the beamtime and to Drs. Vladimir Dmitriev and Raúl Quesada Cabrera for assistance. We thank Dr. Jun Zhao for his assistance with the DSC experiments. B.G. is supported by a Ph.D. grant of the Institute for the Promotion of Innovation through Science and Technology in Flanders (IWT-Vlaanderen). F.M., J.L., S.N., and GVA are postdoctoral research fellows of the Research Foundation Flanders (FWOVlaanderen). The research is funded by the Research Foundation Flanders (G.0214.05) and the Katholieke Universiteit Leuven (OT 03/39).

\section{References and Notes}

(1) McMillan, P. F. J. Mater. Chem. 2004, 14, 1506-1512.

(2) Fabbiani, F. P. A.; Pulham, C. R. Chem. Soc. Rev. 2006, 10, 932-942.

(3) Lu, G. H.; Li, L. G.; Yang, X. N. Macromolecules 2008, 41, 2062-2070.

(4) Wunderlich, B. Thermochim. Acta 1999, 340-341, 37-52.

(5) Burns, G.; Dacol, F. H. Solid State Commun. 1984, 51, 773-775.

(6) Hore, S.; Dinnebier, R.; Wen, W.; Hanson, J.; Maier, J. Z. Anorg. Allg. Chem. 2009, 635, 88-93.

(7) Wunderlich, B.; Jin, Y. Thermochim. Acta 1993, 226, 169-176.

(8) Singh, L. P.; Murthy, S. S. N. Phys. Chem. Chem. Phys. 2009, 11, $5110-5118$.

(9) Dunning, W. J. The crystal structure of some plastic and related crystals. In The plastically crystalline state: Orientationally-disordered crystals; Sherwood, J. N., Ed.; John Wiley \& Sons: Chichester, U.K., 1979; pp 1-37.

(10) Torrisi, A.; Leech, C. K.; Shankland, K.; David, W. I. F.; Ibberson, R. M.; Benet-Buchholz, J.; Boese, R.; Leslie, M.; Catlow, C. R. A.; Price, S. L. J. Phys. Chem. B 2008, 112, 3746-3758.

(11) Granzow, B. J. Mol. Struct. 1996, 381, 127-131.

(12) Geukens, B.; Meersman, F.; Nies, E. J. Phys. Chem. B 2008, 112, 4474-4477.

(13) Hulme, A. T.; Johnston, A.; Florence, A. J.; Fernandes, P.; Shankland, K.; Bedford, C. T.; Welch, G. W. A.; Sadiq, G.; Haynes, D. A.; Motherwell, W. D. S.; Tocher, D. A.; Price, S. L. J. Am. Chem. Soc. 2007, 129, 3649-3657.

(14) Charapennikau, M. B.; Blokhin, A.; Kabo, G. J.; Kabo, A. G.; Diky, V. V.; Gusakov, A. G. Thermochim. Acta 2002, 382, 109-118.

(15) Hammersley, A. P.; Svensson, S. O.; Hanfland, M.; Fitch, A. N.; Häusermann, D. High Pressure Res. 1996, 14, 235-248.

(16) Boultif, A.; Louer, D. J. Appl. Crystallogr. 2004, 37, 724-731.

(17) Favre-Nicolin, V.; Cerný, R. J. Appl. Crystallogr. 2002, 35, 734-743.

(18) Filinchuk, Y.; Talyzin, A. V.; Chernyshov, D.; Dmitriev, V. Phys.

Rev. B 2007, 76, 092104.

(19) Spek, A. Acta Crystallogr. A 1990, 46, C34.

(20) Rodríguez-Carvajal, J. Physica B 1993, 192, 55-69.

(21) Laugier, J.; Bochu, B. LMGP suite for Windows, Laboratoire des Materiaux et du Génie Physique de l'Ecole Supérieure de Physique de Grenoble, http://www.inpg.fr/LMGP.

(22) Bazyleva, A. B.; Kabo, G. J.; Blokhin, A. V. Phys. B-Condens. Matter 2006, 383, 243-252.

(23) Nakamura, N.; Suga, H.; Seki, S. Bull. Chem. Soc. Jpn. 1980, 53, 2755-2761.

(24) Itoh, K.; Shimanouchi, T. Biopolymers 1967, 5, 921-930.

(25) Hexter, R. M. J. Chem. Phys. 1960, 33, 1833-1841.

(26) Schweitzer-Stenner, R.; Sieler, G.; Mirkin, N. G.; Krimm, S. J. Phys. Chem. A 1998, 102, 118-127.

(27) Faurskov Nielsen, O.; Christensen, D. H.; Have Rasmussen, O. J. Mol. Struct. 1991, 242, 273-282.

(28) Smyth, C. P. J. Phys. Chem. Solids 1961, 18, 40-45.

(29) Van Mourik, P.; Veldman, E.; Norder, B.; Van Turnhout, J.; Wübbenhorst, M. J. Mater. Sci. 2005, 40, 1661-1671.

(30) Napolitano, S.; Wübbenhorst, M. J. Non-Cryst. Solids 2007, 353, 4357-4361.

(31) Wübbenhorst, M.; van Turnhout, J. J. Non-Cryst. Solids 2002, 305, 4049.

(32) Starkweather, H. W. Polymer 1991, 32, 2443-2448.

(33) Clemett, C.; Davies., M. Trans. Faraday Soc. 1962, 58, 1718-1728.

(34) Singh, B.; Vij, J. K. Bull. Chem. Soc. Jpn. 1976, 49, 2869-2871.

JP105008K 